\title{
DISTRIBUTIONS ON THE COTANGENT BUNDLE FROM TORSION-FREE CONNECTIONS
}

\section{J. Kurek and W. M. Mikulski}

Summary: Let $m$ and $q$ be arbitrary integers such that $m \geq 1$ and $0 \leq q \leq 2 \mathrm{~m}$. We study the problem how a torsion-free classical linear connection $\nabla$ on an $m$-dimensional manifold $M$ induces canonically a smooth $\left(C^{\infty}\right)$ distribution $A(\nabla) \subset T T^{*} M$ on the cotangent bundle $T^{*} M$ of $M$ such that $\operatorname{dim}\left(A(\nabla)_{\omega}\right)=q$ for any $\omega \in T^{*} M$. We have the following simple examples:

(a) The zero distribution $A^{[1]}(\nabla)$ such that $A^{[1]}(\nabla)_{\omega}=\{0\}$ for any $\omega \in T^{*} M$

(b) The vertical distribution $A^{[2]}(\nabla)=V T^{*} M$;

(c) The full distribution $A^{[3]}(\nabla)=T T^{*} M$;

(d) The $\nabla^{*}$-horizontal distribution $A^{[0]}(\nabla)=H^{\nabla^{*}}$ on $T^{*} M$ (i.e. the horizontal distribution of the linear general connection $\nabla^{*}$ on $T^{*} M \rightarrow M$ dual to $\nabla)$.

We have the decomposition $T T^{*} M=V T^{*} M \oplus H^{\nabla^{*}}$. We recall that given $v \in T_{x} M$ and $\omega \in\left(T^{*} M\right)_{x}, x \in M$, we have the $\nabla^{*}$-horizontal lift $v_{\omega}^{\nabla^{*}}$ of $v$ at $\omega$, i.e. the unique vector from $H_{\omega}^{\nabla^{*}}$ over $v$ with respect to the cotangent bundle projection.

We have the following family of modifications of $H^{\nabla^{*}}$;

(e) Given a canonically dependent on $\nabla$ fibred map $B(\nabla): T^{*} M \rightarrow$ $T^{*} M \otimes T^{*} M$ covering $i d_{M}$, we have the $B(\nabla)$-modification $A^{[B]}(\nabla)$ of $H^{\nabla^{*}}$ such that $A^{[B]}(\nabla)_{\omega}:=\left\{v_{\omega}^{\nabla^{*}}+\left.\frac{d}{d t}\right|_{t=0}(\omega+t<B(\nabla)(\omega), v>) \mid v \in T_{x} M\right\}$ for any $\omega \in\left(T^{*} M\right)_{x}, x \in M$. Then $A^{[B]}(\nabla)$ is a smooth distribution on $T^{*} M$ of dimension $m$ at any point. Clearly, if $B(\nabla)=0$ then $A^{[0]}(\nabla)=H^{\nabla^{*}}$.

We see that $\operatorname{dim}\left(A^{[1]}(\nabla)_{\omega}\right)=0, \operatorname{dim}\left(A^{[2]}(\nabla)_{\omega}\right)=m, \operatorname{dim}\left(A^{[3]}(\nabla)_{\omega}\right)$ $=2 m$ and $\operatorname{dim}\left(A^{[B]}(\nabla)_{\omega}\right)=m$ for any $\omega \in T^{*} M$.

The main result of the present note can be roughly formulated as follows.

Theorem A. All smooth distributions $A(\nabla) \subset T T^{*} M$ on $T^{*} M$ with $\operatorname{dim}\left(A(\nabla)_{\omega}\right)=$ const canonically depending on a torsion-free classical linear connection $\nabla$ on $M$ are the mentioned above distributions $A^{[1]}(\nabla)$, $A^{[2]}(\nabla), A^{[3]}(\nabla)$ and $A^{[B]}(\nabla)$ for fibred maps $B(\nabla): T^{*} M \rightarrow T^{*} M \otimes T^{*} M$ covering $i d_{M}$.

Remark A. One can show (using method from [1]) that the vector space (over $\mathbf{R}$ ) of all $B(\nabla): T^{*} M \rightarrow T^{*} M \otimes T^{*} M$ in question is 3- 
dimensional and the fibred maps $B^{[i]}(\nabla): T^{*} M \rightarrow T^{*} M \otimes T^{*} M$ given by $B^{[1]}(\nabla)(\omega)=\omega \otimes \omega, B^{[2]}(\nabla)(\omega)=\operatorname{sym}\left(\left(\operatorname{Ric}_{\nabla}\right)_{x}\right)$ and $B^{[3]}(\nabla)(\omega)=$ $\operatorname{alt}\left(\left(\operatorname{Ric}_{\nabla}\right)_{x}\right)$ for $\omega \in\left(T^{*} M\right)_{x}, x \in M$, form the basis in this vector space.

\section{References}

[1] Kolář I., Michor P.W., Slovák J., Natural Operations in Differential Geometry, Springer-Verlag Berlin 1993.

[2] Paluszny M., Zajtz A., Foundations of the geometry of natural bundles, Lect. Notes Univ. Caracas, 1984.

Institute of Mathematics

Maria Curie-Sklodowska University

pl. M. Curie-Sklodowska 1, Lublin (Poland)

e-mail: kurek@hektor.umcs.lublin.pl

Institute of Mathematics

Jagiellonian University

Reymonta 4, Kraków, Poland

e-mail: : Wlodzimierz.Mikulski@im.uj.edu.pl 